\title{
Uganda DHIS2 Case Based Surveillance System: Would Have Detected the 2016 Yellow Fever Outbreak in Uganda
}

\author{
Article by Prosper Behumbiize \\ MPH, Health Information Systems Program, Uganda \\ E-mail:ptb3000@gmail.com
}

\begin{abstract}
Majority of developing countries have relayed on aggregate weekly epidemiological data to detect, investigate and respond to outbreak, however as revealed by the West Africa Ebola Outbreak in 2015, these systems were not able to detect the outbreak in time. This has driven countries to building effective case based surveillance systems aimed to link cases to laboratories. Uganda's journey to a web-based electronic case-based surveillance started in 2013 with the United States Government (USG) Uganda Global Health Security (GHS) demo project.

On 24th March 2016, an alert of a suspected Viral Haemorrhagic Fever (VHF) outbreak was received by the Ministry of Health through the Public Health Emergency Operations Centre (PHEOC) and the case was confirmed on 8 April 2016 for Yellow Fever. A total of 60 suspected YF cases were reported between April and June, with 7 cases testing positive.

Building on the successful GHS Specimen Tracking System, we embarked on rebuilding the DHIS2 tracker expanding the scope and coverage to the now eIDSR. Different disease specific Case notification forms were reviewed and common key notification elements harmonized into a general minimum data case DHIS2 tracker registry for immediate reporting.

The system underwent vigorous testing and fine tuning using the yellow fever outbreak cases. The systems demonstrated that it's possible to build an effective case notification and lab confirmation system using DHIS2 tracker with automated SMS and email notification. With the success above it recommended to countrywide rollout and adoption.
\end{abstract}

Keywords: Disease Surveillance, Outbreak, Notifications, Yellow fever, DHIS2, Tracker.

\section{Background}

Majority of developing countries have relayed on aggregate weekly epidemiological data to detect, investigate and respond to outbreak, however as revealed by the West Africa Ebola Outbreak in 2015, these systems were not able to detect the outbreak in time. This has driven countries to building effective case based surveillance systems aimed to link cases to laboratories (Jose and Holman 2017). Uganda's journey to a web-based electronic case-based surveillance started in 2013 with the United States Government (USG) supported through Centers for Diseases Control and Prevent (CDC) Uganda Global Health Security (GHS) demo project. A three-pronged supported was to improve among others information systems to ensure laboratory confirmation is within 24 hours after identification.

Uganda has had a number of outbreaks over the past whose identification and management has not been electronically managed leaving a great impact as result of late confirmation because of the paper data transition and notification from majority of the sources. Implementation of an web-based communicable disease surveillance system (SmiNET-2) in Sweden with immediate case notification and laboratory results revealed the ability for timely case identification, confirmation and outbreak management (Rolfhamre P, Jansson A, Arneborn M, Ekdahl K, 2006).

The purpose of this study was mainly to determine if the newly developed Uganda National Electronic Case Base Surveillance could have timely detected, confirmed and managed the 2016 yellow fever outbreak that was first reported as Viral Haemorrhagic Fever (VHFs) from 3 household members in rural Uganda. 
DOI: $10.21522 /$ TIJAR.2014.04.02.Art016

ISSN: $2520-3088$

The study is both a methodological description of the developed and an evaluation of its capabilities of prevent, detect and respond to infectious diseases.

In 2013, President Obama announced the United States' initiative to support developing countries strengthen their surveillance systems to keep the world safe and secure from infectious diseases. The agenda was endorsed and launched by the G7 countries in February 2014 and now growing with over 50 nations, organizations participating. Prior to its launch the United States Centres for Disease Control and Prevention selected Uganda and Vietnam to demo the initiative by building strengthening surveillance systems around laboratory and information systems. Uganda used the District Health Information Software (DHIS2) to build a case notification, specimen tracking and online lab resulting systems that was piloted in 3 districts during the demo exercises. However, its use and scope expansion did not continue until early 2045 when scale and expansion was realized after the Liberia Ebola outbreak.

Building on the successful Global Health Security DHIS2 Specimen Tracking System, HISP Uganda together with CDC Uganda, MoH Uganda, MoH PHEOC embarked on rebuilding the DHIS2 tracker expanding the scope and coverage to the now eIDSR. This system needed to be stress tested before country rollout and desk testing using the yellow fever data collected both before, within and after the outbreak in 2016.

On 24th March 2016, a phone alert of a suspected Viral Haemorrhagic Fever (VHF) outbreak in Masaka district (Kaloddo village) was received by the Ministry of Health through the Public Health Emergency Operations Centre (PHEOC). Three cases from a single family had presented to Masaka Regional Referral Hospital with high-grade fever that was non-responsive to anti-malarial treatment with haemorrhagic signs and acute neurological signs (convulsions and unconsciousness).

On 8 April 2016, the National IHR Focal Point of Uganda notified WHO of an outbreak of Yellow Fever (YF) in Masaka district, south of Kampala which was confirmed on 8 April 2016 when three blood samples from suspected Viral Hemorrhagic Fever (VHF) cases tested positive for Yellow Fever. A total of 60 suspected YF cases were reported between April and June, with 7 cases testing positive in three districts (5 in Masaka, 1 in Kalangala and 1 in Rukungiri). There was no electronic system in place to support early notification, specimen tracking, online laboratory results entry and outbreak monitoring thus the wide spread and magnitude.

It's evident that lab confirmation of yellow fever took over 24 hours and spread over 3 districts, which given an electronic disease surveillance like the one developed would have helped early detection and management reducing spread.

\section{Methodology}

Building on the Global Health Security Demo specimen tracking systems, the national eIDSR was further customized to address all the immediate notifiable disease in Uganda. A number of different disease specific Case notification forms were reviewed and common key notification elements harmonized into a general minimum data case DHIS2 tracker registry for immediate reporting.

Important laboratory confirmation pieces like test request, specimen tracking and receipt, lab resulting and contact tracing we to be addressed using different DHIS 2 tracker repeated stages accessed and update by different players during detect and confirmation.

The system was presented to different stokeholds including World Health Organization (WHO) who provided valuable feedback to the systems in line with standard disease surveillance. All suggestions and improvements were implemented prior to the evaluation exercise.

The different recent Ugandan outbreaks were evaluated to identify one that could provide sufficient stress testing to the system. Magnitude and spread was key in the selection criteria including availability of data on the cases together with laboratory test results.

Following the order in which cases were identified and reported, data entry of all the cases was carried out from all available Ms Excel and Ms Word line lists and lab focusing on case registration and lab results. 
Data was analysed using the DHIS2 inbuilt analytic tools displaying on dashboards line lists and epicurves. The results were presented to stokeholds involved.

\section{Results and discussions}

\section{System fine-tuning}

Following the yellow fever case definitions and sharing with disease surveillance stakeholder's forum receiving constructive feedback, the electronic system was fine-tuned adding all missing data collections variables and required notifications especially as regards yellow fever identification, confirmation and outbreak management. The email server and SMS getaway were configured to allow for mail and SMS broadcast. Providing an electronic interface for a suspected case registration as seen in Fig 1 below enabled timely notification of the Public Health Emergency Operations (PHEOC) in form of emails and phone short structured messages (SMS).

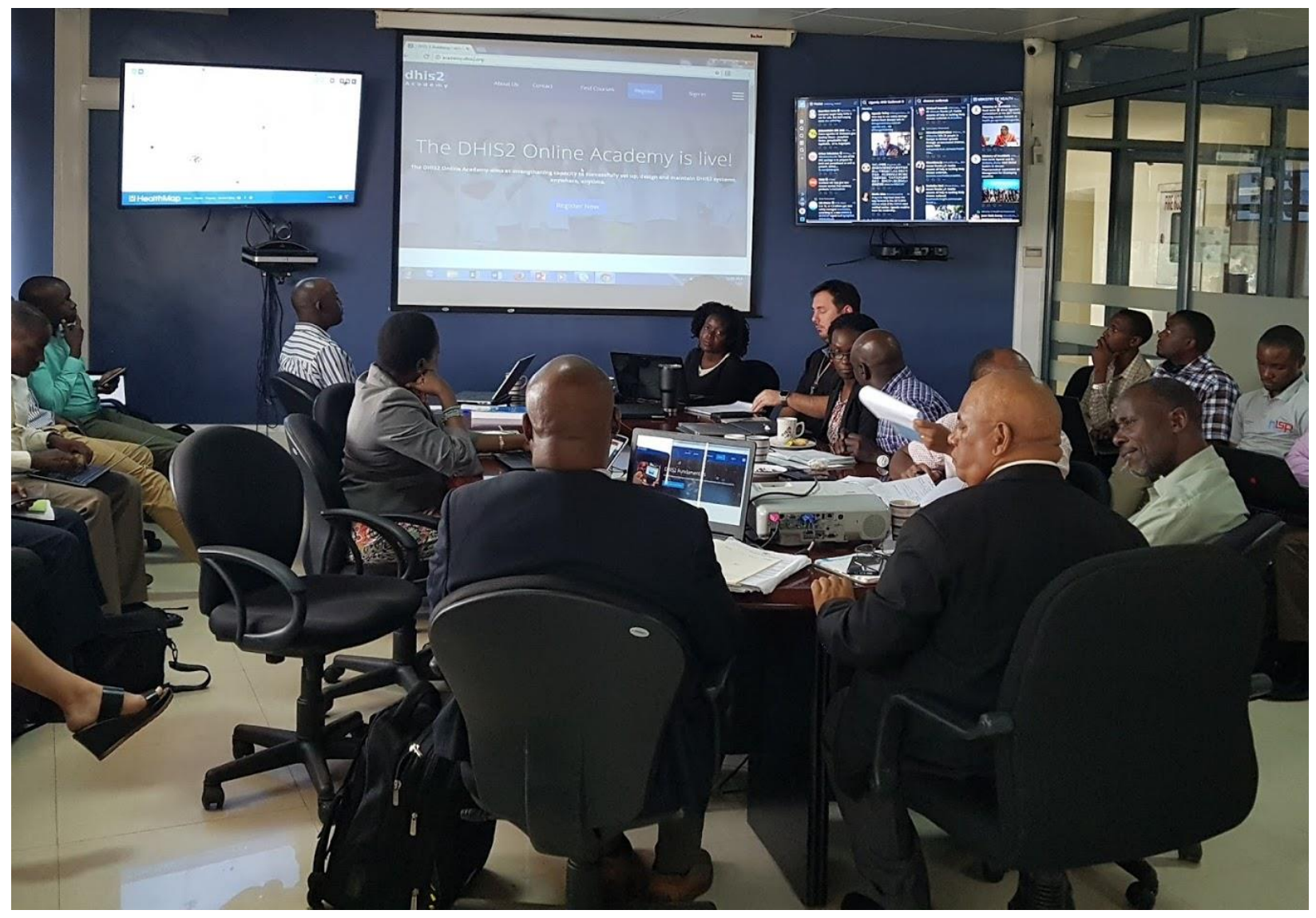

Stakeholder's meeting discussing eIDSR at Ministry of Health Uganda 
DOI: 10.21522/TIJAR.2014.04.02.Art016

ISSN: $2520-3088$

\section{Case Identification, registry and notification}

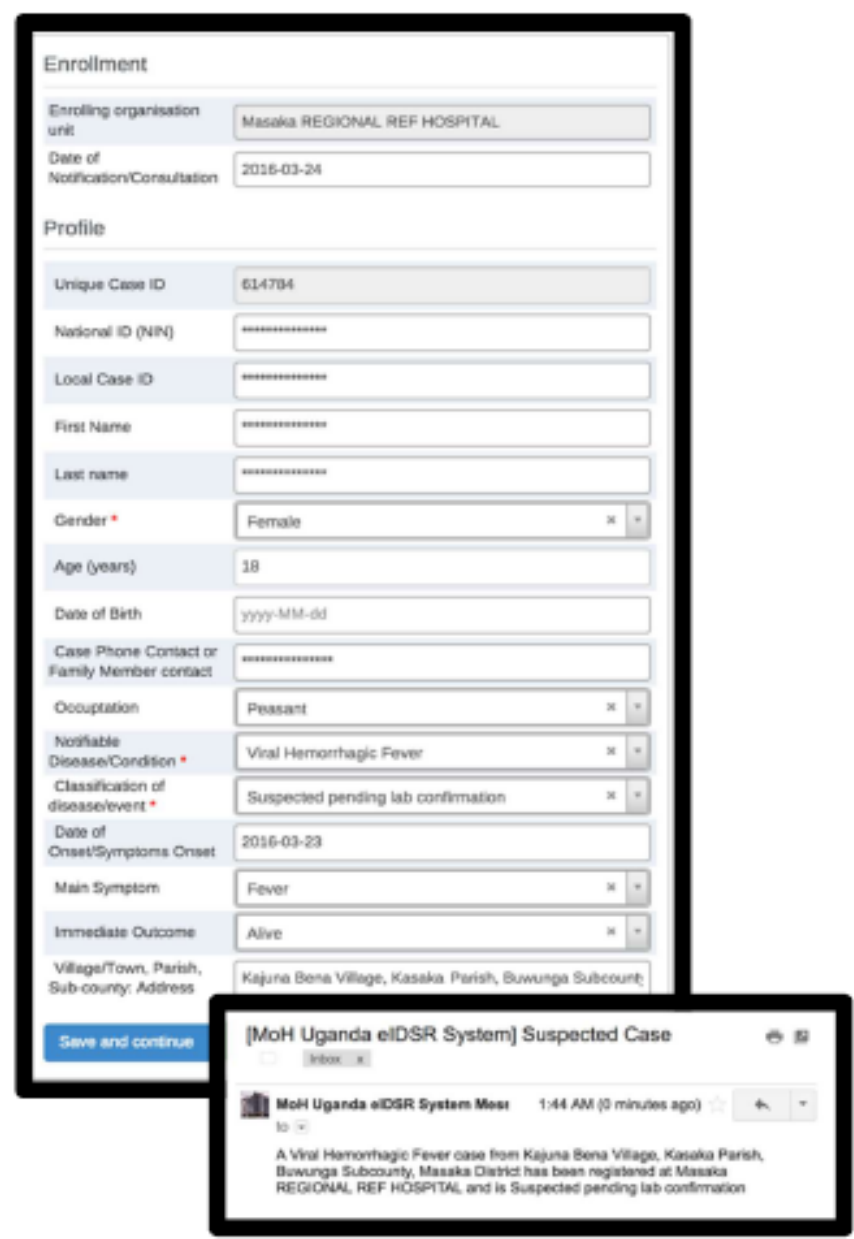

Figure 1. Case registration and notification

On registering the first case, the system was automatically able to send both email and SMS to all National Task Force team members who confirmed by call us back confirming receipt. This created a lot of trust in the system as immediate notification was achieved. Timely notification allows disease surveillance team to quickly swing into action even if it's a rumor. This was a great assurance to the team that as long as the District Disease Surveillance officer has access to the online systems all the members are notified at the same time.

\section{Lab confirmation}

The system allows for laboratory test request while specifying the laboratory to test the specimen, this in term send a notification to the core laboratory team of the specimen referred to them for testing. With the ability to track, the specimen right from the field (source), the testing laboratory received and alert prompting them to start preparing to test the specimen and provide test results within 24 hours. Few hours later assuming the laboratory had received the specimen and tested, online results posted to the system. As illustrated in Figure 2, the entry of lab results trigger an alert the Public Health Emergency Operation Center (PHEOC) and National Task Force, to check the results and proceed with investigations. 


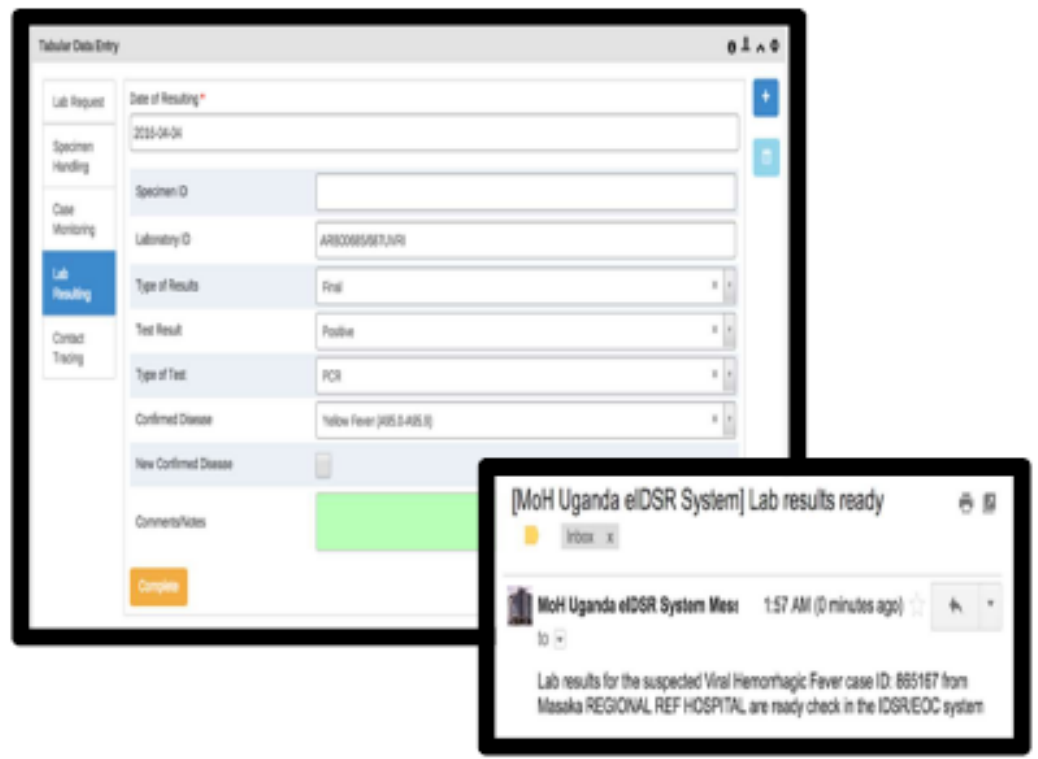

Figure 2. Lab results upload and notification

Both laboratory teams and Task Force teams were able to receive appropriate notification in time to proceed with investigations. The study demonstrated that is possible to build an effective case notification and lab confirmation system using DHIS2 tracker with automated SMS and email notification functionality.

\section{Epidemiology threshold and notification}

WHO recommends countries to setup threshold for each notifiable disease in their setting that would be used to compare with the positive cases for identification of possible outbreaks. For Uganda, any one positive laboratory confirmed case is enough to trigger an outbreak. The system was configured to alert by email and SMS the PHEOC and Task Force members, if one or more cases of Yellow Fever are confirmed from the laboratory tests.

\section{[MoH Uganda eIDSR System] Validation violations as of 2017- $\overline{0}$ 므 07-13T15:59:11_Inbox $x$}

MoH Uganda elDSR System Message [?

10:59 PM (0 minutes ago)

to -

Violations: High 0, medium 1, low 0

Possible Measles Outbreak

The confirmed measles cases 1.0 exceeds the threshold 1.0 from Buikwe $\mathrm{HC} \mathrm{III} \mathrm{for} \mathrm{the}$ period 2017W26. Please investigate

Figure 3. Validation rule notification

Once the systems validations rules run, and the yellow fever cases were one and greater, notifications were sent to the PHEOC and National Task Force members who confirmed receipt. 
DOI: 10.21522/TIJAR.2014.04.02.Art016

ISSN: $2520-3088$

\section{Laboratory testing results}

Samples were collected from all suspected cases and shipped to Uganda Virus UVRI, where testing was done for all possible pathogens including VHF, Chikungunya, Zika and many others. Only 7 cases tested positive for yellow fever. All these laboratory results including the negative pathogen test results were entered into the online system.

With all the data entered, the system customized dashboards were able to display the outbreak Epi-Curve, tool highly used by epidemiologists during an outbreak management to monitor cases daily and weekly. The Epi-Curve as shown in figure 4 below.

Another important outbreak analysis is a line list of all the identified cases for easy contact tracing while conducting further investigation which this system was able to produce this list

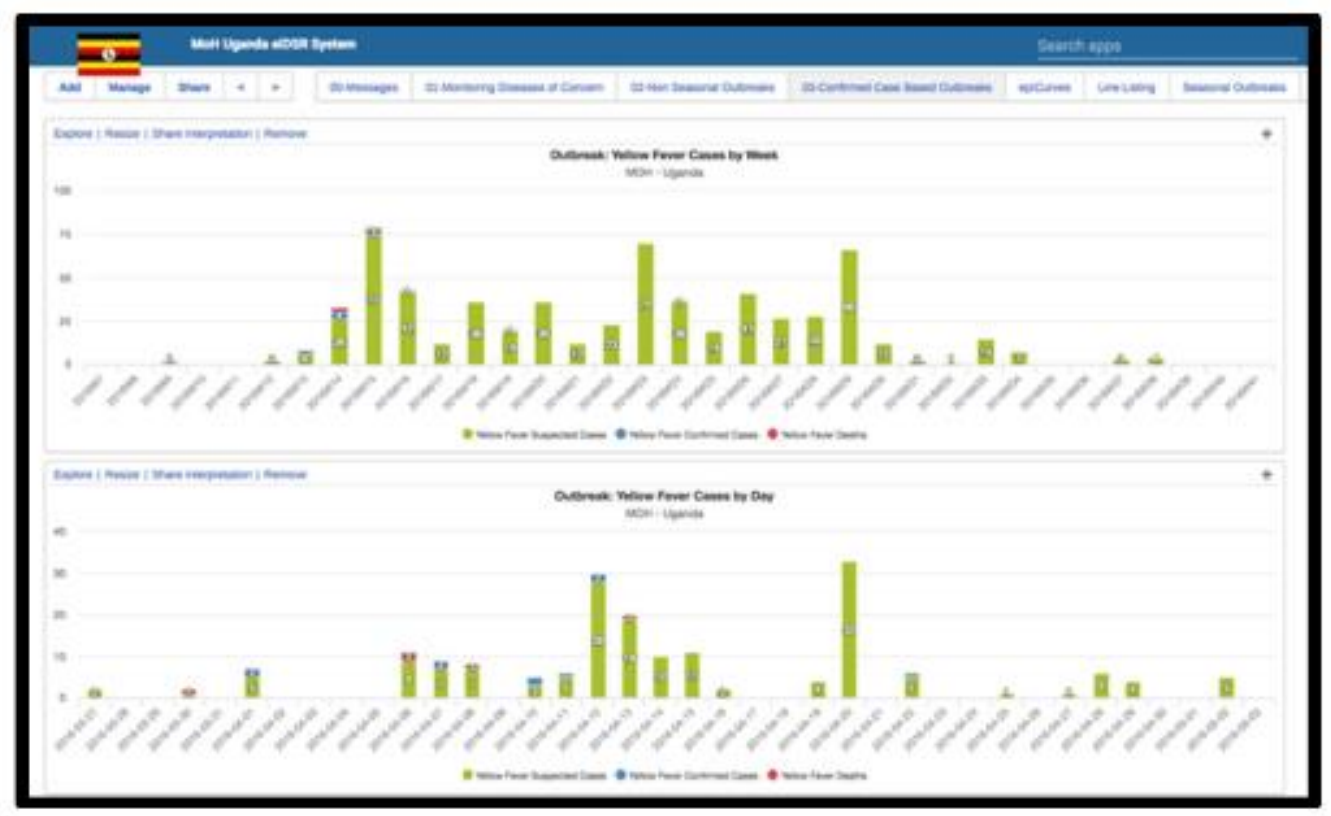

Figure 4. Epi-curve example of yellow fever outbreak 2016

\section{Conclusions}

The ability to replicate the outbreak identification, confirmation and management using retrospective data entry and notifications as described above, clearly demonstrated the capabilities of any disease outbreak containment. The system was also presented to the Uganda eHealth Technical Working Group and approved for rollout. Based on the results and achievements as demonstrated by the study, it's recommended for country rollout to monitor all the Uganda's immediate notifiable diseases.

\section{References}

[1].Jose R, Holman EA, Silver RC. The Importance of the Neighborhood in the 2014 Ebola Outbreak in the United States: Distress, Worry, and Functioning. Health Psychol. 2017 Jul 20. doi: 10.1037/hea0000518.

[2].MOH Uganda. 2015. Integrated Disease Surveillance Action Plan. Unpublished. Ministry of Health, Kampala, Uganda.

[3].MOH Uganda. 2016. Press Statement to Declare End of Yellow Fever Outbreak. Unpublished. Ministry of Health, Kampala, Uganda.

[4].Rolfhamre P, Jansson A, Arneborn M, Ekdahl K, 2006, SmiNet-2: Description of an internet-based surveillance system for communicable diseases in Sweden. Euro Surveillance: Bulletin Europeen sur les Maladies Transmissibles = European Communicable Disease Bulletin [01 Jan 2006, 11(5):103-107].

[5].http://www.who.int/csr/disease/OP_YellowFever_FINAL.pdf: Downolaoded Septemebr, 20th 2017 at 10:00AM 
Texila International Journal of Academic Research Volume 4, Issue 2, Dec 2017

[6].https://www.dhis2.org/ visited on September 19 at 12:00. 